\title{
Esperança e Bem-Estar Psicológico durante a Crise Sanitária pela COVID-19: Estudo com Estudantes de Enfermagem
}

\author{
Hope and Psychological Well-Being during the Sanitary Crisis by COVID-19: A Study with Nursing Students \\ Esperanza y Bienestar Psicológico durante la Crisis Sanitaria por COVID-19: Estudio con Estudiantes de \\ Enfermería
}

Tânia Marlene Gonçalves Lourenço ${ }^{1,2}$ (D)

Zaida Borges Charepe ${ }^{3,4}$ (1)

Cristina Bárbara da Costa Freitas Pestana ${ }^{1}$ [D

Isabel Cristina Mascarenhas Rabiais ${ }^{3}$ (1)

Enrique Jesús Sáez Alvarez ${ }^{5}$ (])

Rita Maria Sousa Abreu Figueiredo $0^{1,2}$ (])

Sérgio Joaquim Deodato Fernandes ${ }^{3,4}$ (D)

1. Escola Superior de Enfermagem São José de Cluny, Gabinete de Investigação e Desenvolvimento Cluny. Funchal, Portugal.

2. Center for Health Technology and Services Research, Innovation \& Development in Nursing Research Group. Porto, Portugal.

3. Universidade Católica Portuguesa, Instituto de Ciências da Saúde. Lisboa, Portugal.

4. Universidade Católica Portuguesa, Centro de Investigação Interdisciplinar em Saúde. Lisboa, Portugal.

5. Universidad Católica de Valencia, Departamento de Enfermagem. Valência, Espanha.
Autor correspondente:

Tânia Marlene Gonçalves Lourenço.

E-mail: tmlourenco@esesjcluny.pt.

Recebido em 28/12/2020.

Aprovado em 26/05/2021.

DOI:https://doi.org/10.1590/2177-9465-EAN-2020-0548

\begin{abstract}
Resumo
Objetivo: analisar as relações entre a esperança e o bem-estar psicológico de estudantes de enfermagem portugueses durante a crise pandêmica pela COVID-19. Método: estudo descritivo-correlacional, com recurso a questionário online para coleta de dados. Participaram 705 estudantes de enfermagem com idade média de 21,74 anos ( $\mathrm{DP}=4,44)$. Os instrumentos de coleta de dados utilizados foram a Escala de Esperança de Herth (HHI-PT) e a Escala de Medida de Manifestação de Bem-Estar Psicológico (EMMBEP). Foi utilizada a estatística descritiva e inferencial. Resultados: na HHI-PT, o domínio afectivo-comportamental obteve a pontuação média mais alta, 3,30 ( $\mathrm{DP}=0,45$ ); no EMMBEP, foi o domínio sociabilidade, 3,64 ( $\mathrm{DP}=0,77)$. Havia correlações moderadas a fortes $(0,383<r<0,917)$ entre o global e todos os domínios da esperança e bem-estar psicológico $(\leq 0.001)$. Conclusão e implicações para a prática: a esperança e o bem-estar psicológico dos estudantes de enfermagem durante a pandemia são variáveis correlacionados positivamente entre si. As medidas de promoção da saúde mental, a implementar pelas instituições de ensino, devem ter em consideração a associação entre essas duas variáveis.
\end{abstract}

Palavras-chave: Esperança; Adaptação Psicológica; Estudantes de Enfermagem; Educação à Distância; COVID-19.

\section{Abstract}

Aim: to analyze the relationship between hope and the psychological well-being of Portuguese nursing students during the COVID-19 pandemic crisis. Method: this is a descriptive-correlational study using an online questionnaire for data collection. 705 nursing students participated, with a mean age of 21.74 years $(S D=4.44)$. The data collection instruments used were the Herth Hope Index (HHI-PT) and the Well-Being Manifestations Measure Scale (WBMMS). Descriptive and inferential statistics were used. Results: in the HHI-PT, the affective-behavioral domain had the highest mean score (3.30; $\mathrm{SD}=0.45)$, while in the WBMMS it was the sociability domain, $3.64(S D=0.77)$. There were moderate to strong correlations $(0.383<r<0.917)$ between the global and all domains of hope and psychological well-being ( $\leq 0.001)$. Conclusion and implications for practice: nursing students' hope and psychological well-being during the pandemic are variables that are positively correlated with each other. Strategies to promote mental health, to be implemented by educational institutions, must consider the association between these two variables.

Keywords: Hope; Psychologic Adaptation; Nursing Students; Distance Education; COVID-19.

\section{Resumen}

Objetivo: analizar la relación entre la esperanza y el bienestar psicológico de los estudiantes portugueses de enfermería durante la crisis pandémica por COVID-19. Método: estudio descriptivo-correlacional mediante cuestionario online para la recogida de datos. Participaron 705 estudiantes de enfermería, con una edad promedio de 21,74 años (DE=4,44). Los instrumentos de recolección de datos utilizados fueron la Escala de Esperanza de Herth (HHI-PT) y la Escala de Medida de Manifestación de Bienestar Psicológico (EMMBEP). Se utilizó estadística descriptiva e inferencial. Resultados: en HHI-PT, fue el dominio afectivoconductual el que obtuvo la puntuación promedio más alta, 3.30 ( $\mathrm{DE}=0.45)$, en EMMBEP, fue el dominio de sociabilidad, 3.64 ( $D E=0.77)$. Hubo correlaciones de moderadas a fuertes $(0.383<r<0.917)$ entre el dominio global y todos los dominios de la esperanza y el bienestar psicológico ( $\leq 0.001)$. Conclusión e implicaciones para la práctica: la esperanza y el bienestar psicológico de los estudiantes de enfermería durante la pandemia son variables que se correlacionan positivamente entre sí. Las medidas de promoción de la salud mental, a ser implementadas por las instituciones educativas, deben tener en cuenta la asociación entre estas dos variables.

Palabras clave: Esperanza; Adaptación Psicológica; Estudiantes de Enfermería; Educación a Distancia; COVID-19. 


\section{INTRODUÇÃO}

A crise pandêmica provocada pela COVID-19, na saúde pública mundial, tem colocado desafios aos domínios científicos da saúde, nomeadamente nas áreas clínicas, políticas, sociais e acadêmicas. Associado a essa realidade e dada as características deste vírus, todos os países têm alternado entre diferentes níveis de contensão social em prol da promoção da saúde pública e proteção de todos, como as atividades implementadas nas instituições de ensino ${ }^{1}$.

A pandemia pode ser entendida como uma crise fortuita no percurso dos estudantes em praticamente todo o mundo. O confinamento obrigatório foi utilizado por vários países, como medida primordial, para conter a disseminação do vírus. A vida em sociedade ficou suspensa, impondo um processo transformativo a todos os níveis e que exigiu às Instituições de Ensino Superior, em particular, reinventarem-se sob pena de paralisarem aquela que é a sua missão: ensinar e aprender. Passar de um processo de ensino-aprendizagem presencial em sala de aula para uma realidade online, com recurso a plataformas eletrônicas, constituiu-se em uma resposta necessária para a continuidade da atividade letiva dos estudantes.

Estudos anteriores à pandemia já identificam os estudantes de enfermagem como um grupo, com níveis moderados a elevados de stress ${ }^{2,3}$, sendo esse um dos preditores de bem-estar psicológico. Também, a esperança já havia sido associada a níveis mais elevados de bem-estar em estudantes universitários ${ }^{4}$. Se, anteriormente à pandemia, já estava disponível essa evidência, a literatura científica pós-COVID-19 tem demonstrado que os estudantes experienciaram alterações do seu bem-estar psicológico com sintomas depressivos, estress e ansiedade durante a crise pandêmica ${ }^{5,6}$.

As circunstâncias de isolamento social e quarentena podem ser experiencialmente vividas como um período de crise nos estudantes, tornando mais complexa e difícil a intervenção em saúde mental. Aos estudantes de enfermagem, acresce a dimensão da natureza do curso; se, por um lado, existe a preocupação com a impossibilidade de retomar os ensinos clínicos e, consequentemente, atrasar a conclusão do mesmo, por outro lado, o retorno aos locais de prática coloca o estudante a um maior risco de exposição ao vírus. Dessa forma, os estudantes de enfermagem devem merecer a nossa particular atenção na gestão do seu bem-estar psicológico e esperança.

A esperança pode ser entendida como uma emoção experienciada por uma atitude pessoal orientada para o futuro ${ }^{7}$ ou uma percepção individual de autoeficácia no cumprimento de metas realistas ${ }^{8}$. Já o bem-estar psicológico, corresponde ao conjunto de recursos emocionais que cada indivíduo dispõe, contribuindo para um funcionamento psicológico positivo 9 .

Mediante o exposto, depreende-se que o conhecimento científico acerca do impacto negativo da pandemia de COVID-19 na saúde mental dos estudantes tem sido francamente desenvolvido, no entanto o enfoque na relação entre a esperança e bem-estar psicológico nesta população é limitado. Neste contexto, estando conscientes do impacto a nível pessoal, familiar, profissional e social, que o ajuste a essa situação de crise de saúde pública exige, realizamos este estudo, que teve como objetivo analisar as relações entre a esperança e o bem-estar psicológico de estudantes de enfermagem portugueses durante a crise pandêmica pela COVID-19.

\section{MÉTODO}

Estudo de natureza quantitativa, do tipo transversal descritivocorrelacional, com recurso a questionário online (Google Forms) para coleta de dados. O link de acesso ao instrumento de coleta de dados foi enviado aos estudantes através de e-mail remetido pela secretaria acadêmica de cada instituição. O estudo foi desenvolvido em seis Instituições de Ensino Superior Portuguesa públicas e privadas. A coleta de dados decorreu no período de 23 a 28 de abril de 2020, altura em que Portugal vivenciava o estado de emergência e, por isso, o confinamento obrigatório em casa. A população-alvo do estudo foi constituída pelos 3003 estudantes a frequentar o Curso de Licenciatura em Enfermagem (CLE). Foi utilizada uma amostra por conveniência, constituída por 705 estudantes inscritos no CLE, nas Instituições de Ensino Superior envolvidas, que aceitaram voluntariamente preencher o questionário online e, por conseguinte, participar no estudo. Enquanto variáveis dependentes, estudaramse a esperança e o bem-estar psicológico.

Foi utilizado o Herth Hope Index, Escala de Esperança de Herth $(\mathrm{HHI}-\mathrm{PT})^{10}$, versão traduzida e adaptada para português europeu. A HHI-PT apresentou uma elevada consistência interna neste estudo, com um coeficiente de alfa de Cronbach de 0,857 , revelando confiabilidade e medindo o que se pretende nesta pesquisa. A HHI-PT é uma escala unidimensional que contém, no total, 12 itens ${ }^{11}$. Cada item está organizado de acordo com uma escala tipo Likert, com uma pontuação variável entre "1"e "4", na qual 1 significa "discordo completamente" e 4 significa "concordo completamente". Essa escala contempla três domínios, correspondentes às subescalas da HHI-PT, designadamente nas dimensões cognitiva-temporal, afetiva-comportamental e afiliativa-contextual da esperança. A dimensão cognitiva-temporal da esperança (sentido interior de temporalidade e futuro, que inclui os itens 1, 2, 6 e 11); a dimensão afetiva-comportamental da esperança (disponibilidade interior positiva e expetativa, que inclui os itens 4, 7, 10 e 12); e a dimensão afiliativa-contextual da esperança (interligação consigo próprio e com outras pessoas, que abrange os restantes itens, a saber os itens 3, 5, 8 e 9) ${ }^{12}$. Nesse instrumento, os itens 3 e 6 apresentam pontuações invertidas, em uma pontuação total variável entre 12 e 48 Quanto maior for a pontuação obtida, maior é o nível de esperança.

A Escala de Medida de Manifestação de Bem-Estar Psicológico (EMMBEP) foi traduzida para português europeu e adaptada para estudantes universitários ${ }^{12}$. O coeficiente de alfa de Cronbach neste estudo foi 0,948 para o global, revelando elevada consistência interna.

A EMMBEP é constituída por 25 itens agrupados em seis domínios: felicidade, sociabilidade, controlo, envolvimento social, autoestima e equilíbrio. Cada item é avaliado em uma escala tipo Likert, que varia de 1 "nunca" a 5 "quase sempre"; a soma de todos os itens resulta em um escore global entre 25-125, em que valores mais elevados correspondem a níveis mais elevados de bem-estar psicológico.

Relativamente às variáveis sociodemográficas, estiveram em estudo as seguintes: gênero, idade, ano curricular em 
frequência no CLE. O questionário também incluiu variáveis relacionados com a experiência de quarentena imposta pela pandemia de COVID-19: dias de quarentena; número de saídas de casa; medo do próprio ou de um familiar ficar infetado. Essa variável foi avaliada através de uma escala tipo Likert, em que o 1 correspondia a "nenhum medo", e 5, a "medo extremamente elevado" de ficar infetado. Também se caracterizou a amostra no que se refere à experiência de ensino à distância através das questões: "Em média, quantas horas por dia permanece no computador, em atividades relacionadas com o ensino à distância (aulas, trabalhos, outros)" e "Nos dias em que tem aulas, em média, quantas horas assiste por dia?".

Avaliou-se, ainda, a satisfação com a vida, pedindo aos participantes que, atendendo às mudanças causadas pela atual pandemia, classificassem a sua vida naquele momento utilizando uma escala de 1 (pior vida possível) a 10 (melhor vida possível).

Antes da coleta de dados, realizou-se um pré-teste ccom 10 estudantes, tendo como finalidade avaliar o tempo despendido no preenchimento, bem como a interpretação das questões colocadas.

Os dados foram tratados com recurso à estatística descritivae inferencial através do software Statistical Package for Social Sciences, versão 26.0. Para a estatística descritiva, recorremos a medidas de tendência central e de dispersão. Na estatística inferencial, usamos vários tipos de análise, como o teste de Kolmogorov-Smirnov com correção Lilliefors, para determinação da normalidade das variáveis, e o coeficiente de correlação de Spearman $(R)$, para determinar a associação entre o bem-estar psicológico e esperança, com as variáveis de caracterização contínuas ou ordinais. $O$ teste $U$ de Mann-Whitney permitiu verificar se havia diferenças entre os valores médios de bem-estar psicológico e esperança entre os estudantes do gênero masculino e feminino. Para identificar se existem diferenças no bem-estar psicológico e esperança entre os estudantes dos diferentes anos do CLE, usou-se o teste Kruskal-Wallis.

A investigação atendeu aos princípios éticos de investigação em ser humanos, nomeadamente, o da autonomia, sendo permitida a recusa em participar. No início do questionário, foi fornecida informação aos participantes sobre o âmbito do estudo, sua natureza, duração e metodologia. $O$ direito à confidencialidade também foi acatado ao longo de toda a pesquisa. Atribuiu-se um número de identificação a cada participante, sendo os dados tratados de forma anônima. Este projeto obteve o parecer favorável da Comissão de Ética para a Saúde da Universidade Católica Portuguesa (Parecer oㅜ 74).

\section{RESULTADOS}

De acordo com a Tabela 1, verificou-se que, dos 705 estudantes de enfermagem, 630 (89,4\%) são do gênero feminino e 75 (10,6\%) são do gênero masculino, com idade média de 21,74 anos $(\mathrm{DP}=4,44)$. Verificou-se que $51,1 \%$ dos estudantes frequentavam ○ $3^{\circ}$ e $\circ 4^{\circ}$ ano do CLE.

A média de dias que os estudantes estiveram em quarentena foi de $44,48(\mathrm{DP}=8,08)$, com uma mediana de saída de casa de 5 vezes. Passavam, em média, 6,5 horas diárias $(\mathrm{DP}=3,40)$ a trabalhar com recurso do computador, das quais, 3,94 horas

Tabela 1. Caraterísticas sociodemográficas dos estudantes de enfermagem ( $n=705)$, Portugal, 2020.

\begin{tabular}{lccccc}
\hline \multicolumn{1}{c}{ Variáveis } & Mín & Máx & M* & DP $^{+}$ & Med $^{\ddagger}$ \\
\hline Idade (anos) & 18 & 52 & 21,74 & 4,44 & 21 \\
\hline Dias de quarentena & 0 & 76 & 44,48 & 8,08 & 45 \\
\hline Saídas de casa durante a quarentena & 0 & 84 & 7,410 & 8,39 & 5 \\
\hline Horas diárias no computador trabalhando & 0 & 18 & 6,50 & 3,40 & 6 \\
\hline Horas diárias de aulas síncronas & 1 & 10 & 3,94 & 1,96 & 4 \\
\hline Medo do próprio ficar infectado (1-5) & 1 & 5 & 3,34 & 1,02 & 3 \\
\hline Medo do familiar ficar infectado (1-5) & 1 & 5 & 4,52 & 0,75 & 5 \\
\hline Satisfação com a vida (1-10) & 1 & 10 & 5,88 & 1,50 & 6
\end{tabular}

Ano do curso $(n=698)$

\begin{tabular}{lll} 
1ㅇano & 205 & 29,4 \\
\hline 2o ano & 136 & 19,5 \\
\hline 3o ano & 183 & 26,2 \\
\hline 4o ano & 174 & 24,9 \\
\hline Gênero & & \\
\hline Feminino & 630 & 89,4 \\
\hline Masculino & 75 & 10,6 \\
\hline
\end{tabular}

$M^{*}$ - média; $D P^{+}$- desvio padrão; $M e^{\ddagger}$ - mediana; $f^{\S}$ - frequência absoluta. 
$(\mathrm{DP}=1,96)$ corresponderam às aulas síncronas assistidas diariamente. Os estudantes têm maior medo de que os seus familiares sejam infetados (média $=4,52: D P=0,75$ ) do que serem eles próprios infetados (média $=3,34: \mathrm{DP}=1,02$ ). A média de satisfação com a vida foi de $5,88(\mathrm{DP}=1,50)$.

De acordo com a Tabela 2, verificou-se que os escores médios dos domínios da escala de bem-estar psicológico são muito próximos, variando entre 3,17 (envolvimento social) e 3,64 (sociabilidade). $\mathrm{O}$ escore médio da escala foi de $3,36(\mathrm{DP}=0,63)$. Os valores do alfa de Cronbach revelaram boa consistência interna em todos os domínios e no global da escala de bem-estar psicológico.

Relativamente à escala da esperança, o domínio afetivocomportamental foi aquele que apresentou escore médio mais elevado, 3,30 ( $D P=0,45)$. O escore médio global da escala foi de $3,13$ ( $\mathrm{DP}=0,42)$. $\mathrm{O}$ alfa de Cronbach dos domínios variou entre 0,597 e 0,763 , sendo 0,857 para o global da escala, revelando resultados aceitáveis de consistência interna.

Nenhum dos domínios, nem os globais, de ambas as escalas, apresentaram uma distribuição normal com $p$-value $\leq 0,001$ no teste de teste de Kolmogorov-Smirnoff com correção Lilliefors.

Através da Tabela 3, constatou-se uma correlação estatisticamente significativa $(p \leq 0,005)$ entre as seguintes variáveis sociodemográficas e o bem-estar psicológico: saídas de casa, horas de trabalho diárias no computador, horas diárias em frequência de aulas síncronas, medo do familiar ficar infectado e satisfação com a vida, sendo esta última uma correlação positiva, moderada e estatisticamente muito significativa $r=0,409(\leq 0,001)$.
Já a correlação entre as variáveis sociodemográficas e a esperança, obteve-se significância estatística com as variáveis: saídas de casa durante a quarentena e satisfação com a vida, sendo esta positiva e moderada $r=0,343(\leq 0,001)$. As variáveis, idade, dias de quarentena, medo do próprio ficar infetado, ano de curso e gênero, não se apresentaram correlacionadas com o bem-estar psicológico e esperança ( $p$-value $>0,05)$.

Da análise da Tabela 4, constata-se que os domínios e globais da escala de bem-estar psicológico e da escala de esperança estão associados entre si, com $p$-values $\leq 0,001 \mathrm{em}$ todas as correlações de Spearman realizadas, sendo essas todas positivas e moderadas a fortes $(, 392<r<, 917)$.

$\mathrm{Na}$ escala do bem-estar psicológico, verificou-se que a felicidade é o domínio que apresenta uma correlação mais forte $(r=0,917)$. Na escala da esperança, é o domínio afiliativocontextual $(r=0,874)$ que apresentou maior correlação.

No bem-estar psicológico, as correlações são mais fortes entre a felicidade e a sociabilidade $(r=0,694)$, entre a felicidade $e$ o controlo $(r=0,675)$, entre o controlo e a autoestima $(r=0,632)$, e entre o equilíbrio e a felicidade. Na correlação entre os domínios das duas escalas, verificou-se que a felicidade e a autoestima, enquanto domínios do bem-estar psicológico, apresentam uma maior intensidade da correlação com a dimensão afiliativocontextual $(0,570<r<0,571)$ da esperança.

O bem-estar psicológico e a esperança dos estudantes de enfermagem durante a quarentena apresentaram uma correlação moderada a forte $(r=0,656, p \leq 0,001)$.

Tabela 2. Análise global das escalas de bem-estar psicológico e de esperança dos estudantes de enfermagem, Portugal, 2020.

\begin{tabular}{|c|c|c|c|c|c|c|c|}
\hline Escalas & Mín & Máx & $\mathrm{M}^{*}$ & $\mathrm{DP}^{+}$ & Med $^{\ddagger}$ & $A C^{\S}$ & K-SII \\
\hline \multicolumn{8}{|l|}{ Bem-Estar Psicológico } \\
\hline \multicolumn{8}{|l|}{ Domínios } \\
\hline Felicidade FE (1-5) & 1,13 & 5,00 & 3,26 & 0,75 & 3,25 & 0,890 & $\leq, 001$ \\
\hline Sociabilidade SC (1-5) & 1,25 & 5,00 & 3,64 & 0,77 & 3,50 & 0,864 & $\leq, 001$ \\
\hline Controlo CT (1-5) & 1,00 & 5,00 & 3,35 & 0,75 & 3,33 & 0,886 & $\leq, 001$ \\
\hline Envolvimento Social ES (1-5) & 1,00 & 5,00 & 3,17 & 0,76 & 3,33 & 0,693 & $\leq, 001$ \\
\hline Autoestima AE (1-5) & 1,00 & 5,00 & 3,35 & 0,72 & 3,25 & 0,817 & $\leq, 001$ \\
\hline Equilíbrio EQ (1-5) & 1,33 & 5,00 & 3,45 & 0,78 & 3,33 & 0,731 & $\leq, 001$ \\
\hline Score médio (1-5) & 1,32 & 5,00 & 3,36 & 0,63 & 3,32 & \multirow{3}{*}{0,948} & \multirow{2}{*}{$\leq, 001$} \\
\hline Global da escala (25-125) & 33,00 & 125,00 & 83,97 & 15,71 & 83,00 & & \\
\hline \multicolumn{7}{|l|}{ Esperança } & \\
\hline \multicolumn{8}{|l|}{ Domínios } \\
\hline Cognitivo-temporal CGT (1-4) & 1,50 & 4,0 & 3,01 & 0,48 & 3,00 & 0,654 & $\leq, 001$ \\
\hline Afectivo-comportamental ACP (1-4) & 1,50 & 4,0 & 3,30 & 0,45 & 3,25 & 0,763 & $\leq, 001$ \\
\hline Afiliativo-contextual AFC (1-4) & 1,25 & 4,0 & 3,09 & 0,50 & 3,00 & 0,597 & $\leq, 001$ \\
\hline Score médio (1-4) & 1,50 & 4,0 & 3,13 & 0,42 & 3,08 & \multirow{2}{*}{0,857} & \multirow{2}{*}{$\leq, 001$} \\
\hline Global da Escala (12-48) & 18,00 & 48,00 & 37,60 & 5,07 & 37,00 & & \\
\hline
\end{tabular}

$\mathrm{M}^{*}$ - média; $\mathrm{DP}^{+}$- desvio padrão; $\mathrm{Med}^{\ddagger}$ - mediana; $\mathrm{AC}^{\S}$ - alfa de Cronbach; K-S"l - teste de Kolmogorov-Smirnov com correção Lilliefors. 
Tabela 3. Correlações entre o bem-estar psicológico e esperança com as caraterísticas sociodemográficas dos estudantes de enfermagem ( $n=705)$, Portugal, 2020.

\begin{tabular}{|c|c|c|}
\hline Variáveis & $\begin{array}{c}\text { Bem-Estar } \\
\text { Psicológico } \\
r(p)^{*}\end{array}$ & $\begin{array}{l}\text { Esperança } \\
\qquad r(p)^{*}\end{array}$ \\
\hline Idade (anos) & $-0,002(0,957)$ & $0,036(0,340)$ \\
\hline Dias de quarentena & $-0,013(0,729)$ & $-0,011(0,762)$ \\
\hline $\begin{array}{l}\text { Saídas de casa durante a } \\
\text { quarentena }\end{array}$ & $0,146(\leq 0,001)$ & $0,122(\mathbf{0 , 0 0 1 )}$ \\
\hline $\begin{array}{l}\text { Horas diárias no computador } \\
\text { trabalhando }\end{array}$ & $-0,089(\mathbf{0}, \mathbf{0 2 8})$ & $-0,009(0,827)$ \\
\hline Horas diárias de aulas síncronas & $-0,089(\mathbf{0}, \mathbf{0 1 8})$ & $-0,031(0,438)$ \\
\hline $\begin{array}{l}\text { Medo do próprio ficar infectado } \\
(1-5)\end{array}$ &,$- 032(0,323)$ & $0,011(0,761)$ \\
\hline $\begin{array}{l}\text { Medo do familiar ficar infectado } \\
(1-5)\end{array}$ & $-0,121(\mathbf{0}, \mathbf{0 1 6})$ & $0,053(0,163)$ \\
\hline Satisfação com a vida (1-10) & $0,409(\leq 0,001)$ & $0,343(\leq 0,001)$ \\
\hline Ano do curso & $p^{+}$ & $p^{+}$ \\
\hline 10 ano & \multirow{4}{*}{0,663} & \multirow{4}{*}{0,529} \\
\hline 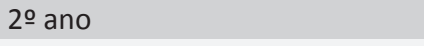 & & \\
\hline 30 ano & & \\
\hline 4 으o & & \\
\hline Gênero & $p^{\neq}$ & $p^{*}$ \\
\hline Feminino & \multirow{2}{*}{0,099} & \multirow{2}{*}{0,149} \\
\hline Masculino & & \\
\hline
\end{tabular}

\section{DISCUSSÃO}

A pandemia está a ter repercussões na saúde mental dos estudantes do ensino superior em geral ${ }^{5} \mathrm{e}$, em particular, nos estudantes de enfermagem ${ }^{6}$. A presente pesquisa identificou que os estudantes que passavam mais horas no computador trabalhando e com maior número de aulas síncronas diárias apresentavam valores estatisticamente significativos mais baixos de bem-estar psicológico $(p \leq 0,05)$. Apesar da rápida adaptação do ensino presencial para o ensino online, a verdade é que as Instituições de Ensino Superior e os seus atores, na sua grande maioria, ainda estavam/estão muito imbuídos e comprometidos com as formas tradicionais de ensino/aprendizagem. Essa transição, que estava prevista surgir naturalmente no ensino da enfermagem ${ }^{13}$, emergiu no centro de uma crise pandêmica, tendo impacto no bem-estar psicológico dos estudantes. Os estudantes participantes neste estudo, à semelhança de outros estudantes universitários, foram confrontados com o imperativo de mudar de paradigma, do ensino presencial para o ensino à distância. Outro resultado que emergiu desta pesquisa foi o número de saídas de casa estar positivamente correlacionado com um aumento, estatisticamente significativo, dos níveis de bem-estar psicológico e esperança dos estudantes $(p \leq 0,05)$. Este achado nos faz refletir sobre os diferentes modelos de confinamento adotados, sendo necessário um equilíbrio entre a manutenção da saúde mental e a salvaguarda da saúde física e a prevenção do risco de infeção em termos coletivos.

Os resultados desta pesquisa revelaram que os estudantes com medo mais elevado de algum do familiar do estudante ficar infetado apresentavam níveis estatisticamente significativos mais baixos de

Tabela 4. Coeficientes de correlação de Spearman entre bem-estar psicológico e esperança de estudantes de enfermagem, Portugal, 2020.

\begin{tabular}{|c|c|c|c|c|c|c|c|c|c|c|c|}
\hline & \multicolumn{7}{|c|}{ Bem-Estar Psicológico } & \multicolumn{4}{|c|}{ Esperança } \\
\hline & $\mathrm{FE}^{+}$ & $\mathrm{SC}^{\ddagger}$ & $\mathrm{CT}^{\S}$ & ES"I & $A E^{\Uparrow}$ & $\mathrm{EQ}^{* *}$ & $\begin{array}{c}\text { Bem-estar } \\
\text { psicológico } \\
\text { global }\end{array}$ & $\mathrm{CGT}^{\ddagger \ddagger}$ & $\mathrm{ACP} \S \S$ & AFC ${ }^{\prime \prime \prime}$ & $\begin{array}{c}\text { Esperança } \\
\text { global }\end{array}$ \\
\hline $\mathrm{FE}^{+}$ & 1 & & & & & & & & & & \\
\hline $\mathrm{SC}^{\ddagger}$ & ,694* & 1 & & & & & & & & & \\
\hline $\mathrm{CT}^{\S}$ &, $675^{*}$ & ,654* & 1 & & & & & & & & \\
\hline ESII &, $527^{*}$ &, $463 *$ &, $473 *$ & 1 & & & & & & & \\
\hline$A E^{\Uparrow}$ & ,682* &, $617^{*}$ &, $632 *$ &, $525^{*}$ & 1 & & & & & & \\
\hline $\mathrm{EQ}^{* *}$ & ,678* & ,613* &, $582 *$ &, $464 *$ &, $581^{*}$ & 1 & & & & & \\
\hline $\begin{array}{c}\text { Bem-estar } \\
\text { psicológico } \\
\text { global }\end{array}$ & ,917* & ,830* & ,799* & ,666* &, $817^{*}$ &, $785^{*}$ & 1 & & & & \\
\hline $\mathrm{CGT}^{\ddagger \ddagger}$ & ,570* &, $466 *$ & ,565* & ,489* &, $571^{*}$ & ,439* & ,629* & 1 & & & \\
\hline $\mathrm{ACP} \S \S$ &, $461^{*}$ & $450 *$ &, $441^{*}$ & ,395* & ,473* & ,383* &, $526 *$ &, $662 *$ & 1 & & \\
\hline AFCIIII &, $526 *$ & $450 *$ & ,450* & ,392* & ,515* &, $421^{*}$ & ,569* &, $617^{*}$ & ,670* & 1 & \\
\hline $\begin{array}{c}\text { Esperança } \\
\text { global }\end{array}$ & ,593* & ,517* & ,552* & ,487* & ,589* & ,473* & ,656* & ,866* & ,872* & ,874* & 1 \\
\hline
\end{tabular}

${ }^{*}$ Correlação significativa para um nível, 01 (2-tailed); $\mathrm{FE}^{\dagger}$ - Felicidade; $\mathrm{SC}^{\ddagger}$ - sociabilidade; $\mathrm{CT}^{\S}$ - controlo; $\mathrm{ES}^{\prime \prime}$ - envolvimento social; $\mathrm{AE}^{\natural}$ - autoestima; $\mathrm{EQ}^{* *}$ equilíbrio; $\mathrm{CGT}^{\ddagger \ddagger}$ - dimensão cognitiva-temporal; ACP ${ }^{\$ \S}$ - dimensão afetiva-comportamental; AFC 1111 - dimensão afiliativa-contextual 
bem-estar psicológico $(p \leq 0,05)$. Os nossos achados vão ao encontro de um estudo realizado com estudantes universitários no Estados Unidos $^{14}$, em que, dos $91 \%$ que revelaram ter medo do próprio ou seu familiar ficar infetado, 1/3 estava ainda mais preocupado, coma saúde dos seus familiares mais vulneráveis, como os idosos ou grávidas.

Ainda, outro estudo, também realizado com estudantes do ensino superior, identificou o medo como um dos preditores da depressão ${ }^{15}$. Por sua vez, da análise realizada em um estudo desenvolvido com jovens adultos na Turquia, foi sugerido o incentivo à partilha de objetivos comuns com outros jovens, à interdependência e sociabilidade como forma de diminuir os níveis de desajuste psicológico ${ }^{16}$. Nesse âmbito, salientamos que, na amostra de estudantes de enfermagem portugueses, os domínios do bem-estar emocional com maiores scores foram precisamente o envolvimento social e o equilíbrio, o que pode conduzir à constatação de que podem ser fatores protetores do bem-estar psicológico em jovens adultos. Esses dados são corroborados por um estudo que identificou o maior suporte social com níveis mais baixo de depressão e maior satisfação com a vida $^{17}$. Relativamente à satisfação com vida, surge, também, na nossa amostra, correlação positiva entre o bem-estar psicológico $(r=0,409 ; p \leq 0,001)$ e a esperança $(r=0,409 ; p \leq 0,001)$.

$O$ percurso no ensino superior per si emerge como um processo de transição múltipla que reforça o estress, desequilíbrios e um ajustamento rigoroso, exigindo uma atenção redobrada para possibilitar um desenvolvimento harmonioso pessoal e profissional do estudante ${ }^{3,4}$, o seu sucesso acadêmico e evitar a morbilidade na vertente da saúde mental.

Apesar de a pandemia se tratar de um fenômeno recente, vários estudos têm demostrado o impacto negativo no bem-estar psicológico dos estudantes ${ }^{5,6,14}$. Os nossos achados apontam para uma média de 83,97 (DP=15,71) na escala de bem-estar psicológico (varia entre 25-125), revelando um nível intermédio.

No que diz respeito aos resultados advindos da avaliação da esperança, o domínio afetivo-comportamental apresentou escores mais elevados. Esse domínio reflete, a partir da perspectiva dos estudantes, um conjunto de sentimentos relacionados com os objetivos que se pretende atingir a percepção de bem-estar, a incerteza de ansiedade, de dúvida, de vulnerabilidade, de preocupação, de raiva, de sofrimento e, por vezes, até de desespero ${ }^{7,11}$.

O presente estudo identificou correlações positivas moderadas a fortes $(0,383<r<0,656)$ entre o global e os vários domínios das duas escalas $(\leq 0,001)$, revelando a associação entre essas duas variáveis. A evidência disponível entre essa associação durante a pandemia ainda é escassa. No entanto, alguns estudos apontam para uma diminuição da ansiedade ${ }^{18}$ e melhoria da saúde mental e bem-estar subjetivo ${ }^{19}$ em indivíduos com níveis mais elevados de esperança, corroborando os nossos resultados.

Os estudantes universitários têm sido identificados como um dos grupos populacionais em risco no que diz respeito ao impacto psicológico da pandemia e pior saúde mental ${ }^{20-22}$. Os estudantes de enfermagem devem merecer a nossa particular atenção na gestão do seu bem-estar psicológico e esperança. A manutenção da saúde mental é fundamental à progressão dos seus objetivos pessoais e acadêmicos, mas também para a futura profissão ${ }^{23}$. Nessa perspectiva, consideramos pertinente ponderar enquanto implicações desta investigação os seguintes aspectos: vulnerabilidade individual, familiar e sociocultural. Todos esses aspectos terão um significativo potencial de acréscimo à vivencia de estress continuado, podendo vir a alterar a percepção dos estudantes quanto ao seu bem-estar psicológico e esperança.

\section{CONSIDERAÇÕES FINAIS E IMPLICAÇÕES PARA A PRÁTICA}

Os achados apontam para uma correlação positiva entre o bem-estar psicológico dos estudantes e a sua esperança durante o confinamento obrigatório resultante da crise pandêmica.

Associado às situações de crise, existe um potencial de crescimento e de desenvolvimento, o qual admitimos poder ocorrer comalguns estudantes. Acreditamos que, para alguns, este será um momento de evolução, de autoconhecimento, de autodescoberta, de aprendizagem, de mudança da visão do mundo, de valorização da comunicação, da relação com o outro, e do que os rodeia, de expressão da humildade, solidariedade, respeito pelo outro, de quebra da cultura do imediatismo, de reflexão e priorização de valores, de um desenvolvimento global edificante enquanto ser humano.

Porém, apesar dos potenciais custos, não ser um cenário de emergência, o melhor contexto para essa mudança paradigmática, o momento pode constituir uma oportunidade para que todos os atores envolvidos nos projetos educativos no nível do ensino superior possam repensar a natureza do conhecimento e dos saberes essenciais a uma sociedade do conhecimento. Estamos convictos de que os resultados obtidos foram influenciados não só pela pandemia em si, mas, também, pelas mudanças ocorridas na transição súbita um ensino à distância, considerando que nem professores nem a maioria dos estudantes estavam preparados para essa transição. O modo como hoje se aprende e qual deverá ser o papel do professor em todo esse processo devem ser atendidos.

Os achados deste estudo poderão ter implicações nas várias áreas da prática em enfermagem. No ensino, poderá ser incluído o componente da esperança e da gestão do medo nos programas de promoção de bem-estar psicológico dos estudantes de enfermagem. $\mathrm{Na}$ pesquisa, os achados apontam para a potencialidade da criação e validação de programas de intervenção que incluam a esperança como preditora do bem-estar psicológico. Ao nível assistencial, os resultados podem nos levar a questionar se essa correlação encontrada em estudantes também não poderá ser encontrada em populações clínicas, abrindo um caminho para a promoção da saúde mental de determinados grupos vulneráveis.

Uma das limitações desta pesquisa poderá ser o tipo e dimensão da amostra, não sendo possível extrapolar os dados para outros estudantes do ensino superior. Também, o fato de ser um estudo descritivo-correlacional impossibilita afirmar uma casualidade entre as correlações estabelecidas. Sugere-se a conceção de estudos similares com amostras maiores que incluam estudantes do ensino superior de outras áreas e, também, a replicação deste estudo em um contexto não pandêmico. 


\section{FINANCIAMENTO}

Este trabalho é financiado por fundos nacionais através da FCT - Fundação para a Ciência e a Tecnologia, I.P., no âmbito do projeto "HumanResponses2COVID-19" (CIIS da UCP).

\section{CONTRIBUIÇÕES DOS AUTORES}

Concepção e desenho do estudo:Tânia Marlene Gonçalves Lourenço. Zaida Borges Charepe. Cristina Bárbara da Costa Freitas Pestana. Isabel Cristina Mascarenhas Rabiais. Enrique Jesús Sáez Alvarez. Rita Maria Sousa Abreu Figueiredo. Sérgio Joaquim Deodato Fernandes

Coleta ou produção de dados: Tânia Marlene Gonçalves Lourenço. Zaida Borges Charepe. Cristina Bárbara da Costa Freitas Pestana. Isabel Cristina Mascarenhas Rabiais. Enrique Jesús Sáez Alvarez. Rita Maria Sousa Abreu Figueiredo. Sérgio Joaquim Deodato Fernandes

Análise de dados: Tânia Marlene Gonçalves Lourenço. Zaida Borges Charepe. Cristina Bárbara da Costa Freitas Pestana. Isabel Cristina Mascarenhas Rabiais. Enrique Jesús Sáez Alvarez.

Interpretação dos resultados: Tânia Marlene Gonçalves Lourenço. Zaida Borges Charepe. Cristina Bárbara da Costa Freitas Pestana. Isabel Cristina Mascarenhas Rabiais. Enrique Jesús Sáez Alvarez.

Redação e revisão crítica do manuscrito: Tânia Marlene Gonçalves Lourenço. Zaida Borges Charepe. Cristina Bárbara da Costa Freitas Pestana. Isabel Cristina Mascarenhas Rabiais. Enrique Jesús Sáez Alvarez. Rita Maria Sousa Abreu Figueiredo. Sérgio Joaquim Deodato Fernandes

Aprovação da versão final do conteúdo do manuscrito a ser publicado: Tânia Marlene Gonçalves Lourenço. Zaida Borges Charepe. Cristina Bárbara da Costa Freitas Pestana. Isabel Cristina Mascarenhas Rabiais. Enrique Jesús Sáez Alvarez. Rita Maria Sousa Abreu Figueiredo. Sérgio Joaquim Deodato Fernandes

Concordância em responsabilizar-se pelos aspectos do trabalho assegurando a acurácia ou integridade de quaisquer parte do trabalho: Tânia Marlene Gonçalves Lourenço.

\section{EDITOR ASSOCIADO}

Antonio José de Almeida Filho (1)

\section{EDITOR CIENTÍFICO}

Ivone Evangelista Cabral (D)

\section{REFERÊNCIAS}

1. Sahu P. Closure of universities due to coronavirus disease 2019 (COVID-19): impact on education and mental health of students and academic staff. Cureus. 2020;12(4):e7541. http://dx.doi.org/10.7759/cureus.7541.

2. Ribeiro F, Mussi FC, Pires C, Silva RMD, Macedo TTS, Santos C. Stress level among undergraduate nursing students related to the training phase and sociodemographic factors. Rev Lat Am Enfermagem. 2020;28:e3209. http://dx.doi.org/10.1590/1518-8345.3036.3209.

3. Labrague LJ, McEnroe-Petitte DM, Gloe D, Thomas L, Papathanasiou IV, Tsaras K. A literature review on stress and coping strategies in nursing students. J Ment Health. 2017;26(5):471-80. http://dx.doi.org/10.1080 /09638237.2016.1244721.
4. Griggs S. Hope and mental health in young adult college students: an integrative review. J Psychosoc Nurs Ment Health Serv. 2017;55(2):2835. http://dx.doi.org/10.3928/02793695-20170210-04.

5. Ma Z, Zhao J, Li Y, Chen D, Wang T, Zhang Z et al. Mental health problems and correlates among 746217 college students during the coronavirus disease 2019 outbreak in China. Epidemiol Psychiatr Sci. 2020 Nov 13;29:e181. http://dx.doi.org/10.1017/S2045796020000931.

6. Reverté-Villarroya S, Ortega L, Lavedán A, Masot O, Burjalés-Martí MD, Ballester-Ferrando D et al. The influence of COVID-19 on the mental health of final-year nursing students: comparing the situation before and during the pandemic. Int J Ment Health Nurs. 2021 Jan 3;30(3):694-702. http://dx.doi.org/10.1111/inm.12827.

7. Cutcliffe JR, Kaye $\mathrm{H}$. The concept of hope in nursing 1: its origins, background and nature. Br J Nurs. 2002;11(12):832-40. http://dx.doi. org/10.12968/bjon.2002.11.12.10307.

8. Snyder CR. The past and possible futures of hope. J Soc Clin Psychol. 2000;19(1):11-28. http://dx.doi.org/10.1521/jscp.2000.19.1.11.

9. Massé R, Poulin C, Dassa C, Lambert J, Bélair S, Battaglini MA Development and validation of a tool for measuring psychological well-being: L'ÉMMBEP. Can J Public Health. 1998;89(5):352-7. http:// dx.doi.org/10.1007/BF03404490.

10. Viana APM. A avaliação da esperança em cuidados paliativos: validação transcultural do Herth Hope Index [dissertação]. Lisboa: Universidade de Lisboa; 2010 [citado 2020 dez 28]. Disponível em: http://hdl.handle. net/10451/2019

11. Herth K. Abbreviated instrument to measure hope: development and psychometric evaluation. J Adv Nurs. 1992;17(10):1251-9. http://dx.doi. org/10.1111/j.1365-2648.1992.tb01843.x.

12. Monteiro S, Tavares J, Pereira A. Portuguese adaptation of the psychological well-being manifestation measure scale with a sample of college students - EMMBEP. Psicol Saude Doencas. 2012;13(1):66-77. http://dx.doi.org/10.15309/12psd130105.

13. Kennedy S. Designing and teaching online courses in Nursing. New York: Springer; 2017.

14. Son C, Hegde S, Smith A, Wang X, Sasangohar F. Effects of COVID-19 on college students' mental health in the United States: interview survey study. J Med Internet Res. 2020;22(9):e21279. http://dx.doi.org/10.2196/21279.

15. Rodríguez-Hidalgo A, Pantaleón Y, Dios I, Falla D. Fear of COVID-19, stress, and anxiety in university undergraduate students: a predictive model for depression. Front Psychol. 2020;11:591797. http://dx.doi. org/10.3389/fpsyg.2020.591797.

16. Satici B, Gocet-Tekin E, Deniz M, Satici S. Adaptation of the fear of COVID-19 scale: its association with psychological distress and life satisfaction in Turkey. Int J Ment Health Addict. 2020;1(9). http://dx.doi. org/10.1007/s11469-020-00294-0.

17. Haliwa I, Spalding R, Smith K, Chappell A, Strough J. Risk and protective factors for college students' psychological health during the COVID-19 pandemic. J Am Coll Health. 2021;(1-5). http://dx.doi.org/10.1080/074 48481.2020.1863413.

18. Mirhosseini S, Dadgari A, Basirinezhad M, Mohammadpourhodki R, Ebrahimi $\mathrm{H}$. The role of hope to alleviate anxiety in covid-19 outbreak among community dwellers: an online cross-sectional survey. Ann Acad Med Singapore. 2020;49(10):723-30. http://dx.doi.org/10.47102/ annals-acadmedsg.2020341

19. Yıldırım M, Arslan G. Exploring the associations between resilience, dispositional hope, preventive behaviours, subjective well-being, and psychological health among adults during early stage of COVID-19. Curr Psychol. 2020. No prelo. http://dx.doi.org/10.1007/s12144-020-01177-2.

20. Moreira WC, Sousa AR, Nóbrega MPSS. Mental illness in the general population and health professionals during COVID-19: a scoping review. Texto Contexto Enferm. 2020;29:e20200215. http://dx.doi. org/10.1590/1980-265x-tce-2020-0215.

21. Maia B, Dias P. Ansiedade, depressão e estresse em estudantes universitários: o impacto da COVID-19. Estud Psicol. 2020;37:e200067. http://dx.doi.org/10.1590/1982-0275202037e200067.

22. Cao W, Fang Z, Hou G, Han M, Xu X, Dong J et al. The psychological impact of the COVID-19 epidemic on college students in China. Psychiatry Res.2020;287:112934. http://dx.doi.org/10.1016/j.psychres.2020.112934.

23. Hughes G, Byrom N. Managing student mental health: The challenges faced by academics on professional healthcare courses. J Adv Nurs. 2019;75(7):1539-48. http://dx.doi.org/10.1111/jan.13989. 